\title{
The Meaning of Weaning: Influence of the Weaning Period on Behavioral Development in Mice
}

\author{
James P. Curley Emily R. Jordan William T. Swaney Asya Izraelit \\ Stella Kammel Frances A. Champagne \\ Department of Psychology, Columbia University, New York, N.Y., USA
}

\section{Key Words}

Weaning - Maternal care $\cdot$ Social behavior - Oxytocin . Vasopressin

\begin{abstract}
Maternal care during the first week postpartum has longterm consequences for offspring development in rodents. However, mother-infant interactions continue well beyond this period, with several physiological and behavioral changes occurring between days 18 and 28 PN. In the present study, we investigate the long-term effects on offspring behavior of being weaned at day 21 PN versus day 28 PN. We found that male and female offspring engage in higher initial levels of social interaction if weaned at day 28 PN, as well as sexually dimorphic changes in exploratory behavior. Females who were themselves weaned earlier also appeared to wean their own pups earlier. Sex-specific effects of weaning age were found on levels of oxytocin and vasopressin V1a receptor density in the hypothalamus, central nucleus of the amygdala and nucleus accumbens. These results indicate that altering weaning age in mice may be a useful model for investigating the development of sexual dimorphism in neurobiology and behavior.
\end{abstract}

\section{KARGER}

Fax +41613061234 E-Mail karger@karger.ch www.karger.com
() 2009 S. Karger AG, Basel
There is converging evidence from numerous laboratory models that experiences occurring early in development can have long-term influences on behavioral and neuroendocrine development. In particular, studies in rodents suggest that variations in the quality of interactions between mother and infant during the postpartum period can induce site-specific changes in gene expression in the brain leading to differences in stress reactivity, social and reproductive behavior, cognition, and rewardmediated behavior [Lehmann and Feldon, 2000; Lippmann et al., 2007; Meaney, 2001]. In these experimental protocols, the quality of the early environment has typically been manipulated through daily separation of mothers from pups for prolonged periods (maternal separation) or brief periods (handling). These manipulations are conducted during the first and second weeks of postnatal development as this is thought to be a critical period for environmentally induced changes in neurobiology. These effects can also be studied in the context of natural variations in care, as both rats and mice display considerable individual differences in maternal behavior during the postpartum period [Champagne et al., 2003, 2007] and these variations are associated with persistent changes in gene expression, physiology and behavior [Meaney, 2001]. However, few studies have explored the
Dr. Frances A. Champagne

Department of Psychology, Columbia University

Room 406 Schermerhorn Hall, 1190 Amsterdam Avenue

New York, NY 10027 (USA)

Tel. +1 212854 2589, Fax +1 212854 3609, E-Mail fac2105@columbia.edu 
influence of maternal care and the quality of the social environment in the later periods of postnatal development. Patterns of maternal care undergo dramatic changes during the postpartum period and mother-infant interactions extend beyond the immediate postnatal period and have been demonstrated in rodents to continue for up to four weeks leading to the phase known as 'weaning' [Konig and Markl, 1987]. This process is marked by a cascade of behavioral and physiological changes in dam and offspring, including a sharp decline in maternal investment and of offspring suckling, the commencement of offspring eating solid food, changes in offspring gut enzyme activity and an increase in offspring socialization and exploration [Martin, 1984]. Thus, weaning should not simply be viewed as an abrupt occurrence, as is often the case in laboratory settings, but is in fact a gradual process occurring over several days with significant behavioral and physiological consequences for offspring [Counsilman and Lim, 1985; Martin, 1984].

Though the transitional nature of the weaning process has been recognized by some developmental biologists and ecologists, in many laboratory environments 'weaning' has become shorthand for the day at which a dam is removed from her litter. Laboratory weaning occurs at various different time-points depending upon the species/strain, ethical regulations and experimental protocols, but typically in the mouse, 'weaning' may occur at any time between 18 and 28 days postnatal (PN). This broad range in animal husbandry practices may be of critical importance when considering the origins of variations in developmental outcomes in rodent studies. The neurobehavioral consequences for laboratory rodents of being removed from their mothers at different ages has primarily focused on the impact of 'early weaning', in which mothers are removed between days 14 and $15 \mathrm{PN}$ ('early-weaned') or between days 20 and 25 PN (labeled as 'normal or standard-weaned'). Studies of Balb/cA and ICR mice weaned from dams at day $14 \mathrm{PN}$ versus day 21 $\mathrm{PN}$, indicate that day $14 \mathrm{PN}$ weaning is associated with altered levels of offspring aggression [Kikusui et al., 2004; Nakamura et al., 2003, 2008], increased anxiety-like behavior [Iwata et al., 2007; Kikusui et al., 2004], increased general activity [Kikusui et al., 2005], and an elevated corticosterone response to stress [Kikusui et al., 2006]. Moreover, females weaned at day $14 \mathrm{PN}$ also exhibited a lower frequency of maternal behavior toward their own pups [Kikusui et al., 2005]. Amongst CD-1 mice weaned from dams at day $15 \mathrm{PN}$ versus day $25 \mathrm{PN}$, day $15 \mathrm{PN}$ weaned offspring were found to exhibit increased locomotor activity [Laviola and Dell'Omo, 1997; Laviola and
Terranova, 1998] and altered social play behavior [Terranova and Laviola, 1995, 2001]. Fewer studies have focused on the neural correlates of these behavioral changes, though mice weaned at earlier time points have been shown to have lower levels of 5HT1B mRNA in the hippocampus [Nakamura et al., 2008], lower brain weights yet higher levels of myelin basic proteins [Kikusui et al., 2007] altered glucocorticoid receptor (GR) mRNA expression [Kikusui et al., 2006], and an increased behavioral sensitivity to the actions of $\delta$-opioid agonists [Terranova and Laviola, 2001] and of D-amphetamine sulfate [Adriani and Laviola, 2002].

These studies illustrate the impact of disruptions or prevention of the weaning process on offspring development. However, the very early time point at which mother-pup separation is imposed in these studies suggest that these results may indicate the effect of maternal deprivation rather than the impact of differences in the natural process of weaning. It could be argued therefore that neither the 'early weaned' nor 'standard weaned' groups in these previous studies have actually undergone weaning at all, but that both have undergone complete maternal deprivation at different time points. In the present study, we explored the process of weaning in C57BL/6J (B6) mice and the influence of experiences during this period of development on offspring neurobiology and behavior. Here we provide the first in-depth analysis of the quality and frequency of mother-infant interactions in B6 mice across 4 weeks of the postnatal period to ascertain whether changes in maternal investment and hence 'weaning' could be observed. Having established that there were indeed significant changes in maternal behavior between day $21 \mathrm{PN}$ and day $28 \mathrm{PN}$ that were congruent with a weaning process, we then chose to investigate the developmental consequences of being weaned at these two time-points on measures of activity and anxiety-like, social and maternal behavior. In addition, we provide the first examination of the influence of social/maternal experiences during this period of development on oxytocin and vasopressin receptor levels in male and female offspring to determine the mechanisms involved in the plasticity in behavioral development that emerge in response to weaning.

\section{Methodology}

Subjects/Procedure

Male and female C57BL/6J (B6) mice of approximately 3 months of age purchased from Charles River were used to generate offspring used in these studies. Mice were housed on a re- 
versed 12-hour dark-light cycle at the Department of Psychology at Columbia University, with lights on at 8 p.m. and off at 8 a.m. All animals were given ad libitum access to food and water. Adult male and female B6 mice were pair housed in same-sex $13.5 \times 8.1$ $\times 5.5$ inch Plexiglas cages in the animal facility for 2 weeks prior to mating. At mating, an adult male was placed with two pairhoused females for a 2 -week period and then removed. Females were monitored daily and singly housed within $48 \mathrm{~h}$ of parturition. On the day of birth $(0 \mathrm{PN})$, pups were weighed and counted and then returned to the home cage. 16 females gave birth and successfully reared pups to weaning. These 16 litters were assigned to be weaned at day $21 \mathrm{PN}(\mathrm{n}=8)$ or day $28 \mathrm{PN}(\mathrm{n}=8)$. These groups are referred to as R21 (removed from dam at day 21 $\mathrm{PN}$ ) and R28 (removed from dam at day $28 \mathrm{PN}$ ), respectively. These litters were observed from PN days 1-6 to determine postnatal levels of maternal care and pups weighed and counted at PN day 6 but otherwise left undisturbed with the exception of weekly cage cleaning until weaning. Additional maternal observations were conducted from PN days 14 to weaning (PN day 21 or PN day 28). At weaning, pups were weighed and counted and then placed 4/cage into same-sex groups with 2 littermates and 2 nonlittermates. From each litter, 2 male and 2 female pups were selected for behavioral testing. A separate sample of 6 male and 6 female pups per group was designated for analysis of receptor binding and was not used for behavioral analysis. Social interaction between cage mates was assessed at day $50 \mathrm{PN}$ and exploratory behavior in the open field and elevated plus maze was determined at day 70 and $74 \mathrm{PN}$, respectively. Females were confirmed to be in diestrus on behavioral testing days using cytological analysis of vaginal swabs. 10 female and 16 male R21 and 16 female and 16 male R28 offspring were tested in the social interaction test. 15 male and 15 female R 21 and 16 male and 16 female R28 offspring were tested in the open field and elevated plus maze. In the elevated plus maze, 2 subjects were eliminated that fell off the maze before the termination of the test. A subset of 8 females per group that were not used in these tests were mated at day 90 PN and those that gave birth ( $\mathrm{n}=7$ R21, n=6 R28) were observed with their litters from days 1 to $28 \mathrm{PN}$. At day $100 \mathrm{PN}$, the offspring designated for receptor analysis were sacrificed and brains extracted for histological preparation. All procedures were conducted in accordance with animal care standards of the Columbia University IACUC.

\section{Maternal Observations}

The procedure for assessing maternal behavior in mice was adapted from studies of natural variations in maternal care in rats [Champagne et al., 2003] and has been described previously [Champagne et al., 2007]. A total of 480 observations of each litter were conducted across each postpartum week. The following behaviors were scored: mother in nursing posture over pups, mother in contact with pups but not in a nursing posture, mother licking and grooming any pup, nest building, self-grooming, eating and drinking. Variations in nursing posture previously observed in lactating rat dams were not evident and nursing thus describes a crouched arch posture over pups. In addition, frequency of 'mounting' behavior was documented in the litters observed from PN days 21 to 28 . This behavior was only observed during the later stages of the preweaning period and is characterized by the dam chasing and pinning the pup while maintaining a rigid thrusting posture over the pups for several seconds. This behavior is typically preceded by repeated attempts by the pup to suckle. Frequency of this behavior was quantified in the R21- and R28weaned offspring.

\section{Social Interaction Test}

On day $50 \mathrm{PN}$, male and female offspring were tested to determine the frequency of social interaction [File and Seth, 2003]. On the day of testing, cage mates were removed from their home cage and housed singly for $10 \mathrm{~min}$ prior to testing. After this short delay, offspring were placed in pairs into a $19.0 \times 10.5 \times 8.0$-inch testing apparatus containing fresh bedding material for $10 \mathrm{~min}$. Following testing, offspring were returned to the home cage. All testing was conducted under dim red lighting. Behavior during the test was recorded with a video camera mounted above the testing apparatus. Time spent sniffing, grooming, mounting and chasing each other during the test was recorded using a stopwatch.

\section{Open Field Test}

The open field apparatus used was a $24 \times 24 \times 16$-inch plastic box. The sides of this box were black and the floor consisted of white tiles. The behavior of the animal in this field was recorded with a video camera mounted on a tripod adjacent to the field. On the day of testing, the mouse was removed from its home cage and placed directly into one corner of the open field. After a 10-min session, the mouse was removed and returned to its home cage. All testing was conducted under red (dark phase) lighting conditions. During analysis of the recordings, the field was divided into a grid of $10 \times 10$ squares. We assessed three behavioral indicators of exploratory behavior: (1) inner field exploration was defined as the time spent in the inner $9 \times 9$ squares, $(2)$ activity was defined as the number of square crossings, and (3) the length of time during which there were no grid crossings was defined as the duration of time spent immobile.

\section{Elevated Plus Maze}

The exploratory behavior of R21- and R28-weaned offspring was measured during a 10 -min elevated plus maze test. The plus maze was elevated 24 inches above the floor on a plastic pedestal. The maze platform and walls consist of black Plexiglas with each 4-inch-wide arm extending 18 inches from the center with 14inch high walls on the closed arms. On the day of testing, the subject animal was removed from the home cage and placed directly in the center of the maze. Behavior during the test was recorded with a video camera mounted over the apparatus. Following testing, the mice were returned to the home cage. Analysis of behavior was conducted using computer software that provides minuteby-minute summary of time spent in the open-arms, closed-arms and center area, as well as the number of entries to the open arms and the number of squares crossings (for the purpose of this analysis the software judges each open and closed arm to be comprised of four squares and the center to be a separate square).

\section{OT and V1a Receptor Autoradiography}

Mice were sacrificed through rapid decapitation and brains extracted, placed briefly in isopentane, and stored at $-80^{\circ} \mathrm{C}$. Brains were sectioned in the coronal plane at $16 \mu \mathrm{m}$, and sections thaw mounted onto poly-L-lysine coated slides that were stored at $-80^{\circ} \mathrm{C}$ until the assay was performed. Slide-mounted coronal brain sections were processed for OT receptor autoradiography 


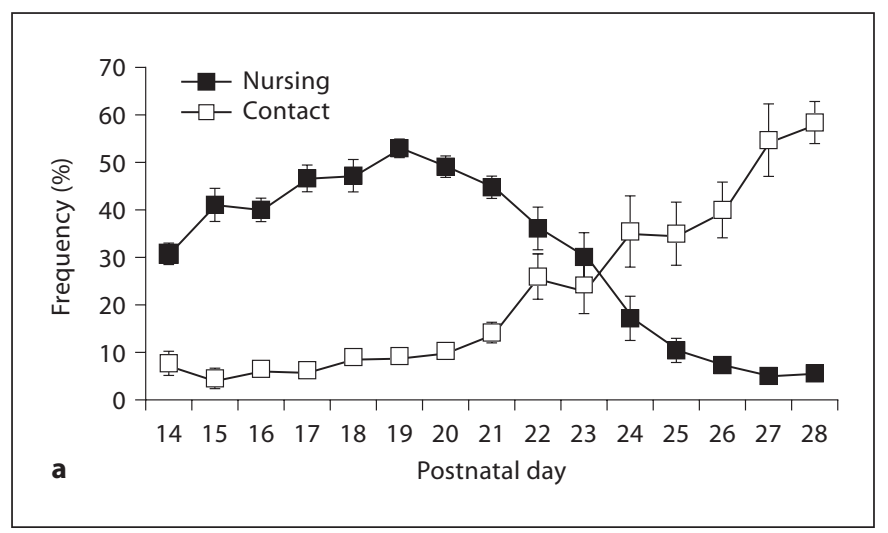

Fig. 1. Frequency of postpartum maternal behavior $( \pm S E M)$ in B6 mice from days $14 \mathrm{PN}$ to $28 \mathrm{PN}$. a Frequency of nursing decreases across this period with increases in the frequency of nonnursing contact. b Maternal licking/grooming (LG) and nest building occur at a low frequency across this period with a slight increase in LG at day 28 PN. Self-grooming increases in frequency across the postpartum period. c Frequency of eating and drinking increase and then decrease as pups switch to eating solid food.

using ${ }^{125} \mathrm{I}-\mathrm{d}\left(\mathrm{CH}_{2}\right)_{5}[\mathrm{Tyr}-\mathrm{Me}]_{2}, \mathrm{Tyr}_{-} \mathrm{NH}_{2}{ }^{9} \mathrm{OVT}$ (New England Nuclear, Boston, Mass., USA) and V1a receptor autoradiography using ${ }^{125} \mathrm{I}$-lin-vasopressin ${ }^{125} \mathrm{I}$-phenylacetyl-D-Tyr(ME)-Phe-GlnAsn-Arg-Pro-Arg-Tyr- $\mathrm{NH}_{2}$ ] (New England Nuclear) as previously described [Francis et al., 2000, 2002]. All autoradiograms were analyzed using an image-analysis system (MC1D-4, Interfocus Imaging, Cambridge, UK). Three sections were analyzed bilaterally for each brain region. For each animal, total and nonspecific binding was measured for each region and the difference taken to yield specific binding. The statistical analysis was performed on the mean of these values for each animal by brain region according to the mouse brain atlas [Paxinos and Franklin, 2003].

\section{Results}

\section{Maternal Behavior during the Postnatal and \\ Preweaning Period}

Observational data were collected from days 1-6 PN (week 1), days 14-21 PN (week 3) and days 22-28 PN (week 4; for R28-weaned litters only). All litters consisted of at least 4 pups with an average litter size of $6.78 \pm 0.49$. Analysis of the frequency of maternal behavior (one-way ANOVA) during these 3 periods indicated a significant
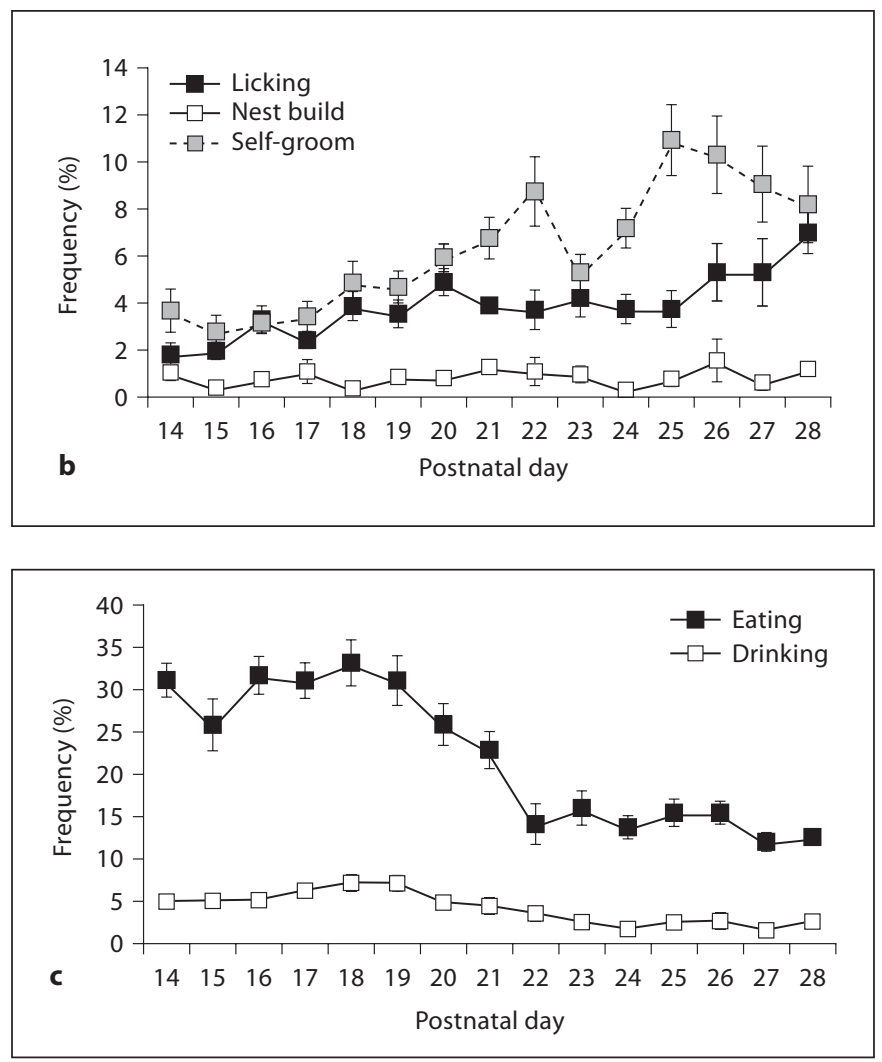

effect of week on frequency of contact $[\mathrm{F}(2,37)=51.30$, $\mathrm{p}<0.001]$, nursing $[\mathrm{F}(2,37)=105.03, \mathrm{p}<0.001]$, licking/ grooming $(\mathrm{LG})[\mathrm{F}(2,37)=28.60, \mathrm{p}<0.001]$, nest building $[F(2,37)=36.74, p<0.001]$, self-grooming $[F(2,37)=$ 53.82, $\mathrm{p}<0.001]$, eating $[\mathrm{F}(2,37)=29.12, \mathrm{p}<0.001]$, and drinking: $[\mathrm{F}(2,37)=47.95, \mathrm{p}<0.001]$. Average frequency of the observed behaviors during each of these postpartum weeks is summarized in table 1 and daily average frequencies of behavior during weeks 3 and 4 are presented in figure 1 . Frequency of non-nursing contact was found to increase significantly in postnatal week 4 ( $\mathrm{p}<$ $0.001)$ corresponding to significant decrease in nursing contact in weeks 3 and 4 ( $\mathrm{p}<0.001)$. Likewise, there is a significant decline in frequency of LG and nest building in weeks 3 and $4(\mathrm{p}<0.001)$ compared to the first week postpartum. Self-grooming was found to increase steadily during the postpartum period, with highest levels observed in postnatal week 4. Frequency of eating and drinking were found to be highest during week 3 of the postpartum period with significant decreases in the frequency of these behaviors during week 4 . 
Fig. 2. Duration of social interaction in seconds ( \pm SEM) amongst R21- and R28weaned male and female offspring. Duration of social interaction during the first minute of testing was higher amongst R28weaned offspring (a), whereas total duration of social interaction during the 10min test did not differ as a function of weaning age (b). ${ }^{*} \mathrm{p}<0.05$.

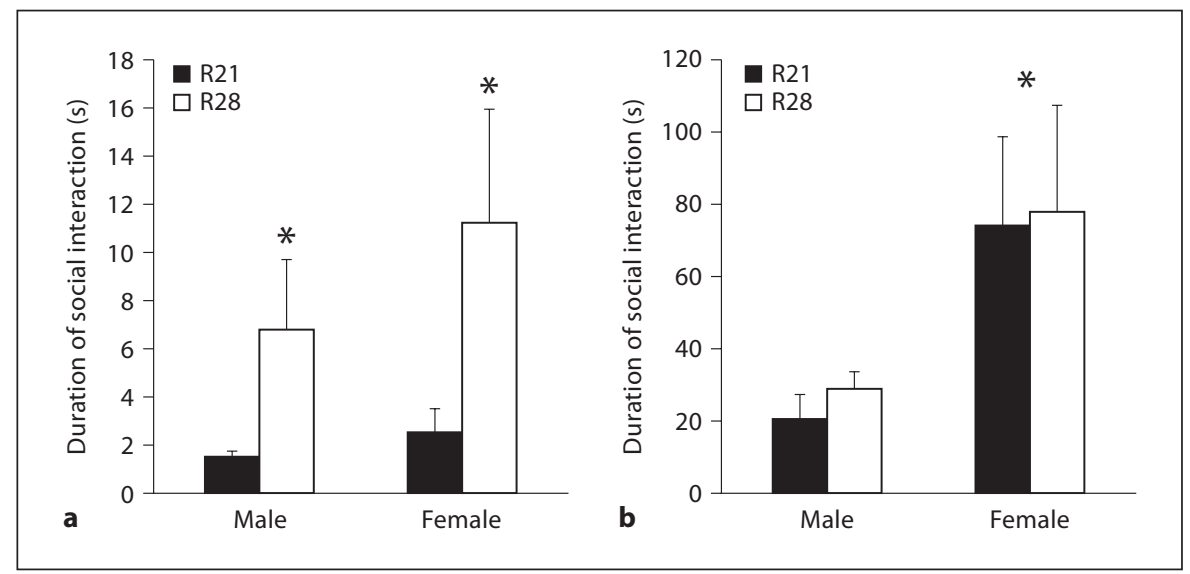

Table 1. Variation in maternal behavior during the postpartum period

\begin{tabular}{|c|c|c|c|c|c|c|c|}
\hline & Contact & Nursing & Lick/groom & Nest build & Self-groom & Eating & Drinking \\
\hline Week $1(n=16)$ & $8.78 \pm 0.65$ & $56.46 \pm 1.62$ & $7.90 \pm 0.57$ & $5.39 \pm 0.62$ & $3.00 \pm 0.26$ & $19.78 \pm 0.86$ & $2.09 \pm 0.16$ \\
\hline Week $3(n=16)$ & $8.72 \pm 1.02$ & $44.25 \pm 1.39$ & $3.28 \pm 0.26$ & $0.84 \pm 0.13$ & $4.46 \pm 0.29$ & $29.25 \pm 1.65$ & $5.66 \pm 0.38$ \\
\hline Week $4(n=8)$ & $37.34 \pm 5.24$ & $16.51 \pm 2.68$ & $4.53 \pm 0.61$ & $0.90 \pm 0.26$ & $8.62 \pm 0.63$ & $14.35 \pm 0.89$ & $2.44 \pm 0.25$ \\
\hline
\end{tabular}

Table 2. Characteristics of postnatal environment of R21- and R28-weaned litters

\begin{tabular}{|c|c|c|c|c|c|c|c|}
\hline $\begin{array}{l}\text { Weaning } \\
\text { age }\end{array}$ & $\begin{array}{l}\text { Litter size } \\
\text { at day } 0 \mathrm{PN}\end{array}$ & $\begin{array}{l}\text { Pup weight } \\
\text { at day } 0 \mathrm{PN}, \mathrm{g}\end{array}$ & $\begin{array}{l}\text { Litter size } \\
\text { at day } 6 \mathrm{PN}\end{array}$ & $\begin{array}{l}\text { Pup weight } \\
\text { at day } 6 \mathrm{PN}, \mathrm{g}\end{array}$ & $\begin{array}{l}\text { Nursing days } \\
1-6 \text { PN, \% }\end{array}$ & $\begin{array}{l}\mathrm{LG} \\
\text { days 1-6 PN, \% }\end{array}$ & $\begin{array}{l}\text { Pup weight } \\
\text { at weaning, g }\end{array}$ \\
\hline $\mathrm{R} 21$ & $9.00 \pm 0.42$ & $1.25 \pm 0.03$ & $7.50 \pm 0.33$ & $3.20 \pm 0.13$ & $54.62 \pm 1.97$ & $7.83 \pm 0.96$ & $7.78 \pm 0.27$ \\
\hline $\mathrm{R} 28$ & $8.62 \pm 0.62$ & $1.27 \pm 0.03$ & $7.87 \pm 0.48$ & $3.31 \pm 0.18$ & $56.23 \pm 2.53$ & $8.42 \pm 0.63$ & $12.28 \pm 0.46^{* * *}$ \\
\hline
\end{tabular}

Litter Size, Weight and Mother-Infant Interactions in

R21- and R28-Weaned Mice

Comparison of litter size and average pup weight at birth (day $0 \mathrm{PN}$ ) and at day $6 \mathrm{PN}$ indicated no significant differences between litters selected for R21 versus R28 weaning (table 2). Likewise, frequency of maternal care during the first week postpartum received by R21- and R28-weaned offspring did not differ. Weaning weight was significantly elevated amongst R28- compared to R21-weaned offspring [ $\mathrm{t}(14)=8.79, \mathrm{p}<0.001$; table 2].

\section{Social Interaction by R21- and R28-Weaned Mice}

Two aspects of social interaction were analyzed: (1) initial behavior within the 1st minute of testing, and (2) overall duration of social interaction during the 10 - min test. The scores obtained for each dyad were analyzed as independent units of data. Two-way ANOVA with weaning age and sex as factors indicated a main effect of weaning age on duration of initial social interaction $[\mathrm{F}(1,25)=4.85, \mathrm{p}<0.05]$ but no main effect of sex or a significant weaning age by sex interaction. R28-weaned male and females spent significantly more time engaged in social interaction during the first minute of testing than R21 animals (fig. 2a). Analysis of total duration of social interaction during the test indicated a main effect of sex $[\mathrm{F}(1,25)=7.12, \mathrm{p}<0.05]$ but no main effect of weaning age and no weaning age by sex interaction. Females were found to engage in a higher duration of social interaction with each other compared to males regardless of weaning age (fig. 2b). 


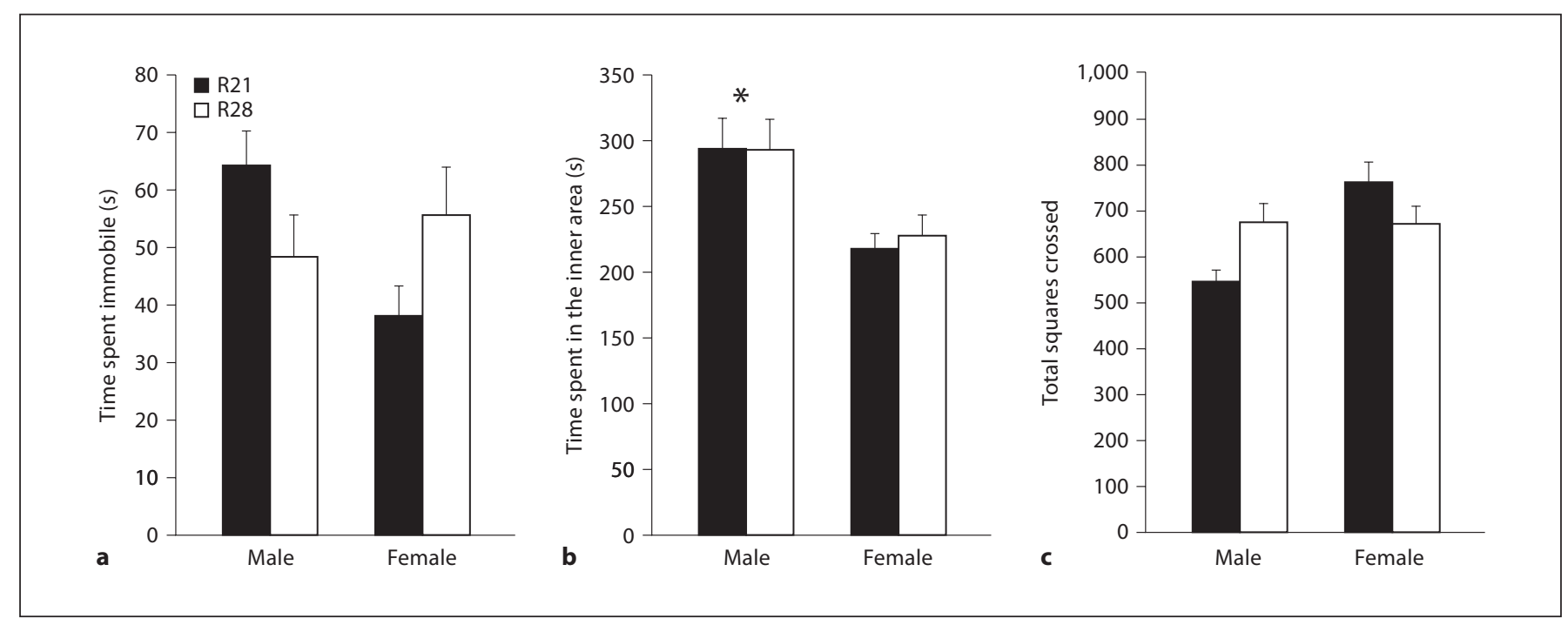

Fig. 3. Open-field behavior of R21- and R28-weaned offspring a Time spent immobile ( \pm SEM) was found to be decreased in R21-weaned females compared to R21-weaned males with no sex differences in R28-weaned offspring. $\mathbf{b}$ Time spent in the inner area of the field ( \pm SEM) was not altered as a function of weaning age. c Total squares crossed during testing ( \pm SEM) indicated sex differences in R21-weaned offspring, with females more active than males. Consistent with immobility findings, there were no sex differences in activity amongst R28-weaned offspring. ${ }^{*} \mathrm{p}<$ 0.05 .

\section{Exploratory Behavior of R21- and R28-Weaned Mice in the Open Field}

Two-way ANOVA indicated no main effect of weaning age or sex on time spent immobile with a significant weaning age by sex interaction $[\mathrm{F}(1,58)=5.82, \mathrm{p}<0.05]$. Tukey's post-hoc analysis indicated sex differences in time spent immobile (with females spending less time immobile than males) amongst R21-weaned ( $\mathrm{p}<0.05)$ but not R28-weaned offspring (fig. 3a). Analysis of time spent in the inner area indicated a main effect of sex $[\mathrm{F}(1,58)=13.80, \mathrm{p}<0.001]$ with no effect of weaning age nor a weaning age by sex interaction. Regardless of weaning age, females spent less time in the inner area of the field compared to males (fig. 3b). We found a significant main effect of sex $[\mathrm{F}(1,58)=8.06, \mathrm{p}<0.01]$ and a significant weaning age by sex interaction $[\mathrm{F}(1,58)=8.86$, $\mathrm{p}<0.01]$ on total grid crossings during the 10 -min test. Similar to the analysis of immobility, post-hoc analysis indicated a sex difference in grid crossings (with females observed to engage in more grid crossings than males) amongst R21-weaned offspring ( $\mathrm{p}<0.001$ ) but no sex differences amongst R28-weaned offspring (fig. 3c). A significant main effect of sex was found on number of grid crossings in the outer area of the field $[\mathrm{F}(1,58)=$ $13.89, \mathrm{p}<0.001]$ and a weaning age by sex interaction $[\mathrm{F}(1,58)=9.67, \mathrm{p}<0.01]$ on number of grid crossings in the inner area. Females were found to engage in more grid crossings in the outer area of the field regardless of weaning age and R28-weaned males were found to engage in an increased number of grid crossings in the inner area compared to R21-weaned males ( $\mathrm{p}<0.05)$.

\section{Exploratory Behavior of R21- and R28-Weaned Mice in the Elevated Plus Maze}

Analysis of behavior during the 10-min session indicated a main effect of sex on time spent in the open-arms $[\mathrm{F}(1,56)=5.21, \mathrm{p}<0.05]$, closed-arms $[\mathrm{F}(1,56)=7.12, \mathrm{p}<$ $0.01]$ and center area $[F(1,56)=7.17, p<0.01]$ but no significant effects of weaning age on these measures (table 3). Females were found to spend more time in the closed-arms than males $(486.19 \pm 6.09$ s vs. $455.18 \pm$ $10.03 \mathrm{~s})$ and less time in the open-arms $(75.76 \pm 4.86 \mathrm{~s}$ vs. $97.91 \pm 8.68 \mathrm{~s})$ and center area $(38.05 \pm 2.14 \mathrm{~s}$ vs. $46.91 \pm 2.44 \mathrm{~s}$ ) regardless of weaning age. As we found that the R21 and R28 groups varied in their degree of sexual differentiation in the open-field behavior, we performed $t$ tests on the total time spent in each arm between males and females within each group. In R28weaned mice, sex differences were found in time spent in the closed-arms $[\mathrm{t}(28)=2.33, \mathrm{p}<0.05]$ and center area $[\mathrm{t}(28)=3.03, \mathrm{p}<0.01]$ with a trend for time spent in the open-arms area $[\mathrm{t}(28)=1.88, \mathrm{p}=0.07]$. When open-arms 
Table 3. Behavior of mice in an elevated plus maze over a 10 -min period

\begin{tabular}{lcccc}
\hline Behavior & R21 males & R21 females & R28 males & R28 females \\
\hline Total time in closed-arms, s & $464 \pm 12$ & $484 \pm 7$ & $445 \pm 17$ & $488 \pm 10$ \\
Total time in open-arms, s & $89 \pm 11$ & $74 \pm 4$ & $107 \pm 14$ & $77 \pm 8$ \\
Total time in center area, s & $46 \pm 3$ & $42 \pm 4$ & $48 \pm 4$ & $35 \pm 2$ \\
Number of open-arm entries & $24.6 \pm 1.6$ & $22.1 \pm 1.7$ & $23.6 \pm 1.9$ & $19.8 \pm 1.2$ \\
Total number of square crossings & $279.1 \pm 23.6$ & $298.0 \pm 16.4$ & $291.0 \pm 33.5$ & $257.4 \pm 10.9$ \\
Number of square crossings in closed-arms & $193.4 \pm 18.5$ & $214.6 \pm 12.4$ & $202.1 \pm 26.7$ & $183.2 \pm 8.6$ \\
Number of square crossings in open-arms & $26.7 \pm 2.3$ & $28.0 \pm 2.0$ & $28.4 \pm 3.1$ & $26.8 \pm 2.5$ \\
Number of crossings (center area) & $58.9 \pm 4.2$ & $68.3 \pm 12.7$ & $60.4 \pm 5.5$ & $47.4 \pm 2.6$ \\
\hline
\end{tabular}

and center area time was combined these sex differences were also significant $[\mathrm{t}(28)=2.33, \mathrm{p}<0.05]$. R28 females spent more time than R28 males in the closed-arms, and less time in the open-arms or center area. No such sex differences were observed between R21 males and females.

Two-way ANOVA revealed that males of both groups made significantly more open-arm entries than females $[\mathrm{F}(1,56)=3.94, \mathrm{p}<0.05]$, with no main effect of weaning age (table 3). When examining each sex independently, there were still no sex differences between R21 males and females in their open-arm entries, but a trend for R28 males to enter the open-arms more frequently than R28 females $[t(28)=1.80, p=0.08]$. In addition, the total number of square crossings made by subjects was calculated as a measure of activity levels. R28 females made significantly fewer total crossings in all arms than did R21 females [ $\mathrm{t}(28)=2.11, \mathrm{p}<0.05]$, which was specifically related to these females making fewer crossings within the closed-arms area $[\mathrm{t}(28)=2.12, \mathrm{p}<0.05]$ as there was no difference in the number of crossings made in the openarm area. There were no differences in the total number of crossings made between R21 and R28 males. No sex differences were found in total activity in either the openor closed-arms areas between R21 males and females or R28 males and females, but R28 males were found to make significantly more crossings through the center area $[\mathrm{t}(28)=2.24, \mathrm{p}<0.05]$ than $\mathrm{R} 28$ females, which was an effect not found in R21 animals.

Maternal Behavior of R21- and R28-Weaned Females

We found no significant effects of weaning age on litter size or average pup weight of pups born to R21-weaned $(\mathrm{n}=7)$ or R28-weaned $(\mathrm{n}=6)$ females. Repeated-measures analysis indicated significant variations in the frequency of all maternal behaviors across the 4 weeks post- partum [contact: $\mathrm{F}(3,11)=53.51, \mathrm{p}<0.001$; nursing: $F(3,11)=32.13, p<0.001$; LG: $F(3,11)=7.98, p<0.05$; nest building: $F(3,11)=15.78, p<0.001$; self-grooming: $F(3,11)=17.41, p<0.001$; eating: $F(3,11)=12.72, p<0.001$; drinking: $F(3,11)=13.87, p<0.001]$. However, there were no significant effects of weaning age on the frequency of these behaviors (though there was a trend for R21-weaned females to spend more time in non-nursing contact with offspring in week 4; fig. 4a). Analysis of the onset and frequency of pup mounting was found to differ between R21- and R28-weaned females. R21-weaned females were observed to have an onset of mounting behavior from days 18 to $23 \mathrm{PN}$ whereas for R28-weaned females the onset ranged from PN days 21 to $27 \mathrm{PN}$. Comparison of average day of onset of mounting indicated a trend $[t(11)=2.02, p=0.068]$ for R21-weaned females to have an earlier day of onset than R28-weaned females (R21weaned: day $21.14 \pm 0.59$; R28-weaned: day $23.17 \pm$ $0.83)$. Repeated measures ANOVA indicated a main effect of day $[F(1,11)=2.60, p<0.01]$ and a main effect of weaning age $[\mathrm{F}(1,11)=17.03, \mathrm{p}<0.01]$ on the frequency of mounting behavior from days 18 to $28 \mathrm{PN}$. Posthoc analysis revealed that $\mathrm{R} 21$-weaned females were observed to engage in higher levels of this behavior at days 22-23 $\mathrm{PN}$ ( $\mathrm{p}<0.05$; fig. $4 \mathrm{~b})$.

\section{OT and V1a Receptor Binding in R21- and}

R28-Weaned Mice

Density of OT and V1a receptors in adult R21- and R28-weaned offspring was compared in the central nucleus of the amygdala (CA), bed nucleus of the stria terminalis (BNST), lateral septum (LS), medial preoptic area (MPOA), nucleus accumbens core (NAc), nucleus accumbens shell (NAs) and ventral medial hypothalamus (VMH) (fig. 5). Two-way ANOVA indicated no significant effects of sex or weaning age on OT receptor density 

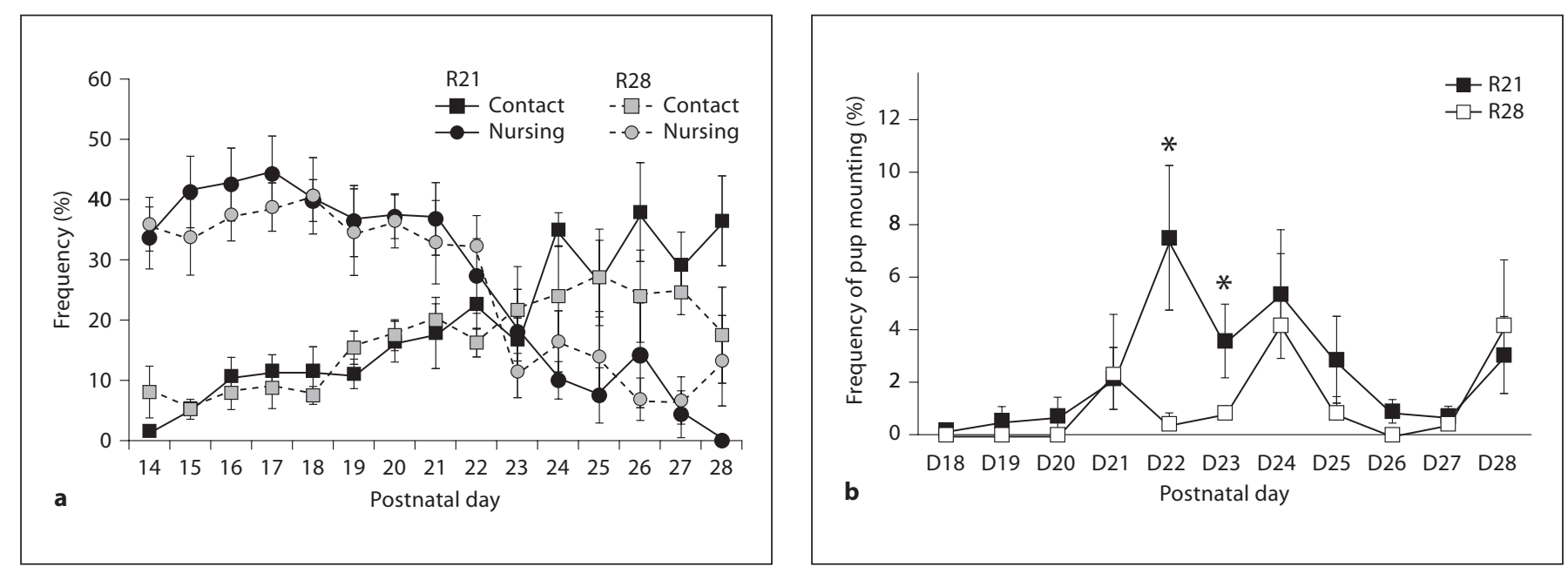

Fig. 4. Maternal behavior of R21- and R28-weaned offspring. a Frequency of nursing and non-nursing contact ( \pm SEM) during days 14-28 PN, which diverge more significantly in R21 females after day 21 PN. b Frequency of pup mounting ( \pm SEM) from days 18-28 PN. R21-weaned females were observed to engage in significantly higher levels of this behavior at days $22 \mathrm{PN}$ and $23 \mathrm{PN} .{ }^{*} \mathrm{p}<0.05$.

in the CA. Analysis of V1a binding indicated a main effect of sex on receptor density in the CA $[\mathrm{F}(1,16)=4.72$, $\mathrm{p}<0.05$ ] with elevated V1a receptor binding in females compared to males. Sex differences in V1a receptor binding were apparent in R21-weaned mice, with females having higher CA binding than males $[\mathrm{t}(8)=2.63, \mathrm{p}<0.05]$ but no sex differences existed in R28 animals. Within the BNST, we found no significant effects on OT receptor binding yet a significant effect of weaning age on V1a receptor binding $[\mathrm{F}(1,16)=4.51, \mathrm{p}<0.05]$ with elevated V1a receptor density in R28-weaned offspring. Post-hoc analysis indicated that R28-weaned females had higher V1a receptor binding than $\mathrm{R} 21$ females $[\mathrm{t}(8)=2.53, \mathrm{p}<0.05$ ] but no effect of weaning age was found on V1a receptor binding in males. Within the MPOA there was no significant group effects on OT receptor binding and a significant weaning age by sex interaction on $\mathrm{V}$ la receptor density $[\mathrm{F}(1,16)=13.45, \mathrm{p}<0.01]$. Post-hoc analysis indicated that there was a significant increase in V1a receptor density in the MPOA of R28-weaned females compared to both R21 females and R28 males ( $<<0.05)$. No significant effects of sex or weaning age were found on OT or V1a receptor density in the LS or the NAc and NAs. However, there was a trend for higher OT receptor binding in R28 females compared to R21 females in the NAs $[\mathrm{t}(8)=2.23, \mathrm{p}=0.057]$. Within the $\mathrm{VMH}$, we found a significant effect of sex $[\mathrm{F}(1,16)=17.9, \mathrm{p}<0.001]$ and a significant sex by weaning age interaction $[\mathrm{F}(1,16)=7.07$, $\mathrm{p}<0.05$ ] on OT receptor binding. Post-hoc analysis indicated sex differences in OT receptor binding in R28weaned offspring and no sex-differences amongst R21weaned offspring. This effect was due to decreases in OT receptor binding in R28-weaned males and increases in OT receptor binding in R28-weaned females.

\section{Discussion}

Overall, these results illustrate the developmental influence of weaning age and suggest that experiences during the later postnatal period can have significant influences on sexual dimorphism in brain and behavior. Though previous studies of weaning age have primarily used a model in which pups are removed from the dam at very early time points, a manipulation that is more similar to those used in maternal separation paradigms, here we demonstrate that extending the duration of contact between mother and siblings can induce long-term changes in the exploratory, social, and maternal behaviors of offspring. Taken together with the patterns of maternal behavior we observe across the postpartum period, these results suggest that changes in the pattern of maternal care may be a critical feature of the weaning process and that the experience of this transition has significant implications for offspring development in a sex-specific manner. 


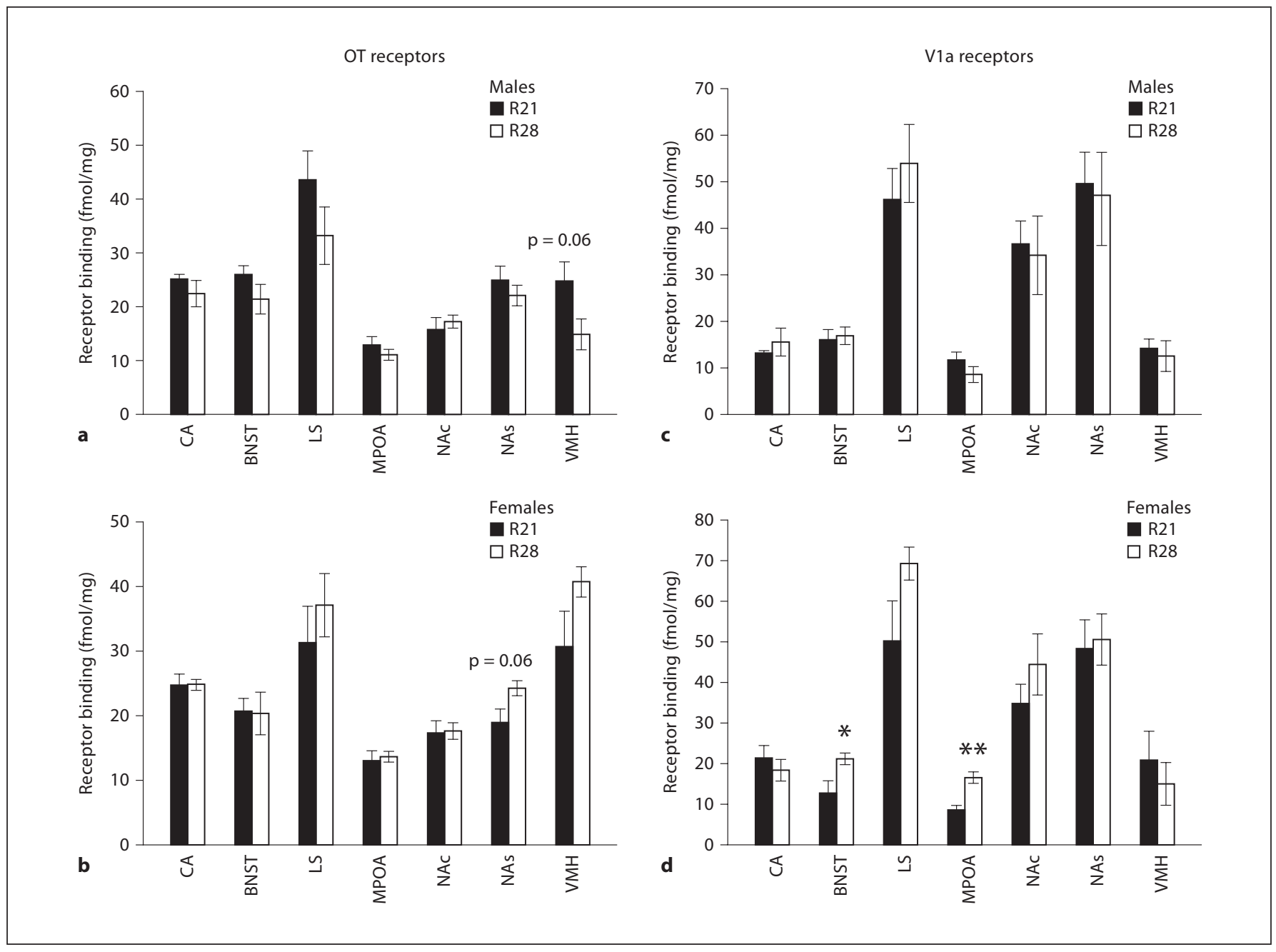

Fig. 5. Receptor autoradiography measurement of OT and V1a receptor density (fmol/mg \pm SEM) in the in central nucleus of the amygdala (CA), bed nucleus of the stria terminalis (BNST), lateral septum (LS), medial preoptic area (MPOA), nucleus accumbens core (NAc), nucleus accumbens shell (NAs) and ventral medial hypothalamus (VMH). Comparison of OT receptor density in (a) R21- and R28-weaned males, and (b) R21- and R28-weaned females. V1a receptors were also compared in R21- and R28-weaned males (c) and females (d). ${ }^{*} \mathrm{p}<0.05,{ }^{* *} \mathrm{p}<0.001$.

\section{Variations in Maternal Behavior across the}

Postpartum Period: The Meaning of Weaning?

Few studies have thoroughly investigated the patterns of maternal care exhibited by mouse dams over 28 postnatal days. In our initial cohort of B6 females, the highest frequencies of nursing were observed immediately postpartum and then continued to steadily decline throughout the first 3 weeks postpartum. Congruent with these changes, the frequency of time spent in contact with pups without nursing increased following day $21 \mathrm{PN}$ from 14\% to $30 \%$ by day $24 \mathrm{PN}$. Throughout the final postpartum week, the frequencies of nursing and contact with pups without nursing continued to diverge demonstrating that the weaning period was terminating (fig. 1a). The frequency of time that lactating dams spent eating and drinking corresponded to the nutritional demands of pups, with decreases in these behaviors associated with the transition of pups to solid food (fig. 1c). These patterns observed in lactating B6 mice are consistent with previous reports of postpartum behavior in wild house mice (Mus domesticus) [Konig and Markl, 1987] and with studies of inbred mouse lines [Benus and Rondigs, 1996] 
indicating dramatic reductions in nursing behavior on day $23 \mathrm{PN}$.

In the present study, we provide the first analysis of mother-pup mounting behavior in rodents. This behavior was observed most often immediately after pups would seek nipple contacts, would continue for several minutes during which time a female would switch her mounting between different pups. Though we were not able to discern whether male or female pups were mounted more frequently, this would certainly be interesting to investigate further given that other forms of tactile stimulation such as maternal LG have previously been demonstrated to be preferentially given to males in the latter periods of lactation and to perhaps lead to the development of sex differences in behavior [Birke and Sadler, 1987; Moore, 1984]. Given that female mounting behavior is often considered to be related to the establishment of dominant-subordinate relationships [Fang and Clemens, 1999], and that this behavior was observed immediately following nipple contact solicitations by pups, it appears as if maternal mounting may be an active component of the weaning process undertaken by the dams. To our knowledge, there are no other published reports of such a behavior occurring in mice dams though few studies have explored mother-infant interactions during this period. Overall, our observations of a decline in nursing, the onset of pup mounting, increases in non-nursing mother-pup contact, and the gradual switch from milk to eating of solid foods by pups, suggest that the weaning process in mice is a long gradual transition between days 18 and $28 \mathrm{PN}$. The question raised is how disruption of this process, which is likely the norm rather than the exception in most laboratory settings, alters the development of offspring.

\section{Weaning Age Influence on Anxiety-Like, Exploratory} and Social Behavior

One of the primary outcome measures in studies of early maternal deprivation, exposure to low levels of maternal care, and deprivation of maternal contact through early weaning are changes in anxiety-like behavior. We find that R21 and R28 males and females do not differ from each other on standard measures of anxiety-like behavior such as time in the inner area of the open-field or time spent in the open- versus closed-arms in the elevated plus maze. However, R21 females did demonstrate elevated levels of activity during behavioral testing whereas R21 males were found to be less active than R28 males. As a consequence of these divergent effects in males and females on measures of exploratory behavior, we ob- served effects on sexual dimorphism as a function of weaning age. These data suggest that the additional week of maternal care and sibling contact received by R2 8 animals leads to females developing a less active and exploratory phenotype whereas males develop a more active and exploratory phenotype.

Previous studies of the effects of weaning at day $14 \mathrm{PN}$ suggest that the removal of the mother at this time-point is associated with increased anxiety-like behavior as evidenced by fewer open-arm entries, more closed-arm entries, less time spent in the open-arms and more time in the closed-arms of an elevated plus maze compared to mice weaned at day 21 PN [Iwata et al., 2007; Kikusui et al., 2004, 2005, 2006]. Furthermore, offspring of both sexes weaned at earlier time points have been shown to exhibit increased general activity levels as juveniles and in adulthood [Kikusui et al., 2005; Laviola and Dell'Omo, 1997; Terranova and Laviola, 1995, 2001; Terranova et al., 1998]. In rats, similar increases in anxiety-like behavior and activity have been observed in early-weaned offspring [Kanari et al., 2005; Shimozuru et al., 2007] though males appear to be more affected than females [Ito et al., 2006; Kodama et al., 2008]. Though the magnitude of the effect is somewhat lessened, these differences have also been reported amongst rat pups weaned at day $21 \mathrm{PN}$ compared to those weaned at day $30 \mathrm{PN}$ [Ferdman et al., 2007; Joffe and Levine, 1973]. As with the mouse, the weaning process in the rat is also gradual [Smith, 1991], with nursing behavior still occurring up to day $33 \mathrm{PN}$. Thus, it appears as if the sudden removal of rodent pups from the natal environment before they are able to complete the gradual transition characteristic of weaning leads to these offspring developing an increase in general activity as well as anxiety-like behavior similar to other animals who also experience periods of maternal separation early in life [Lehmann and Feldon, 2000; Lehmann et al., 1999]. However, more subtle changes seem to occur to animals that are removed from their mother at later periods of development, with sex-specific effects emerging.

During the juvenile period of development, we find that R28 males and females engage in higher levels of initial social interaction when placed with a familiar samesex peer. Previous studies have shown a similar increase in social investigation in animals that have had experienced extended periods of maternal care. In 25- to 30day-old mice, animals weaned at day $25 \mathrm{PN}$ engaged in a longer duration of olfactory investigation of same-sex and age mice in comparison to offspring weaned at day 15 PN [Terranova and Laviola, 2001]. In adult rats, ani- 
mals weaned at day $30 \mathrm{PN}$ compared to day $21 \mathrm{PN}$ were found to spend more time in contact and in social investigation with a novel same-sex animal [Ferdman et al., 2007]. Conversely, rats weaned at day 14 PN have been shown to exhibit reduced frequencies of play fighting compared to animals weaned later [Janus, 1987; Shimozuru et al., 2007], though some evidence suggests that during the very early juvenile period, rat offspring weaned at earlier time points may show an increase in play behaviors [Brunelli et al., 1989; Smith, 1991]. As the decrease in social investigation that we observe in R21 weaned offspring is limited to the first minute of contact, these weaning age effects may relate to a decrease in social neophobia among animals who have undergone the process of weaning through extended mother-infant contact. This interpretation is consistent with previous literature which has used social behavior in a novel setting such as the social interaction test as an indicator of anxiety-like behavior [File and Seth, 2003].

\section{Weaning Age and Maternal Behavior: Transmission} from Mother to Daughter

The transmission of variations in maternal behavior from one generation to the next has been demonstrated across numerous species. In rodents, female offspring who receive low levels of maternal LG in the early postnatal period are observed to engage in low levels of this form of maternal care when they are adults and caring for their own offspring [Champagne et al., 2003; Francis et al., 1999]. Similar mother to daughter transmission is observed on measures of attachment and parental bonding in humans [Miller et al., 1997; Sroufe, 2005] and frequency of physical contact with infants and maternal rejection in primates [Berman, 1990; Fairbanks, 1989; Maestripieri, 2005]. However, less is known about how variations in parental care received later during the postpartum period may shape the future parental behaviors exhibited by rodent offspring. We found no differences between R21 and R28 females in their frequencies of nursing, LG, nest building or any nonmaternal behaviors during the first 3 weeks postpartum.

In contrast, previous studies involving weaning of mice or rats at day $14 \mathrm{PN}$ suggest that maternal deprivation at this earlier period of development results in reductions in nursing and LG by female offspring [Kikusui et al., 2005; Skolnick et al., 1980]. However, we do find evidence that weaning age significantly alters behaviors that emerge in the later postpartum period. R21 females were observed to spend an increased amount of time resting by their offspring without nursing late in the fourth week postpartum and a decreased frequency of nursing on day 28 PN compared to R28 females (fig. 4a). This increased divergence between the frequencies of non-suckling contact and nursing has been demonstrated to occur following the conclusion of weaning in altricial rodents [Konig and Markl, 1987]. Moreover, R21 animals showed their sharpest decline in nursing at day $21 \mathrm{PN}$ whilst this occurred at day $22 \mathrm{PN}$ in R28 animals, which also suggests that R21 females are weaning their offspring earlier than R28 females [Martin, 1984]. Earlier weaning is also demonstrated by the earlier onset and increased frequency of pup mounting following pup suckling solicitations by R21 females during week 4 of the postpartum period. Thus, females who are themselves weaned earlier attempt to wean their own offspring earlier, suggesting that mother-infant experiences beyond the immediate postpartum period may influence the transmission of maternal care across generations. Moreover, these data illustrate that the nature of the effect of early life experience on maternal care will be dependent on the timing during postnatal development when these experiences occur.

\section{Neurobiological Changes Associated with Weaning}

Age: Plasticity of OT and Vla Receptor Pathways

Neuropeptide receptors for oxytocin and vasopressin have previously been demonstrated to regulate social, maternal and anxiety-like behaviors in rodents [Donaldson and Young, 2008; Keverne and Curley, 2004]. Weaning age and sex were not found to influence the density of oxytocin receptors in the CA, BNST, LS, MPOA, or NAc suggesting that variations in OT receptor density may not account for observed behavioral changes in exploratory and social behavior [Keverne and Curley, 2004]. However, in the VMH, a region commonly identified as regulating sex differences in socio-sexual behaviors [Cushing and Kramer, 2005; Dugger et al., 2007], we find sex differences in OT receptor binding in R28 but not R21 mice, due to an increase in OT receptor binding in R28 females and a decrease in OT receptor binding in R28 males. In females, estradiol-induced increase in OT receptor expression within the $\mathrm{VMH}$ is critical for the regulation of sexual behavior by oxytocin [Bale et al., 2001; McCarthy et al., 1994]. Although we did not measure sexual behavior in these offspring, given the observed receptor differences in the $\mathrm{VMH}$, it is possible that variations in weaning age may well induce differences in female sexual behavior. Low levels of maternal care experienced during the early postnatal period have previously been associated with increased lordosis quotients of female offspring corresponding to altered hypothalamic estro- 
gen receptor (ER) expression [Cameron et al., 2008a; Cameron et al., 2008b]. In males, the VMH is critical in the regulation of sexual and aggressive behaviors primarily through the actions of estrogen on ER $\alpha$ [Harding and McGinnis, 2004; Nelson and Trainor, 2007]. OT receptor expression in the $\mathrm{VMH}$ is up-regulated following increases in estradiol [Bale et al., 2001] or decreases in progesterone [Windle et al., 2006], and estrogen and oxytocin receptor mRNA is found to be co-expressed in $\mathrm{VMH}$ neurons [Devidze et al., 2005]. Taken together with our data, these findings suggest that variation in maternal care across the whole of the postnatal period can induce changes in offspring reproductive strategies. The increased mounting behavior observed amongst R21weaned females may also suggest an effect on socio-sexual behavior, possibly driven by hypothalamic neuropeptide receptor levels.

Previous studies of the role of vasopressin in the regulation of social affiliation, aggression and anxiety have largely focused on the regulation of male behavior. For instance, altered social behavior is found in male but not female V1a receptor knockout mice (KO) and V1a receptor $\mathrm{KO}$ males show reduced anxiety during elevated plusmaze testing [Bielsky et al., 2004, 2005; Egashira et al., 2007]. However, there is recent evidence that vasopressin is involved in mediating maternal care. For instance, V1a receptor antagonists chronically infused either centrally or into the MPOA lead to reduced levels of maternal aggression, nursing and LG of pups [Bosch and Neumann, 2008; Nephew and Bridges, 2008]. Moreover, upregulation of V1a receptors in the MPOA through use of viral vectors is associated with increased levels of nursing and LG of pups [Bosch and Neumann, 2008]. Though the influence of vasopressin on maternal behavior in the later postpartum period has not yet been explored, it is possible that the behavioral differences observed amongst R21 versus R28 lactating females during week 4 may be associated with the alterations in hypothalamic V1a receptor density in conjunction with the reduction of OT receptor density observed in $\mathrm{R} 21$ females in the $\mathrm{VMH}$.

Our findings contribute to accumulating evidence that variations in social experience are able to induce long-term changes in the expression of oxytocin and vasopressin receptors. Previous work has illustrated the effects of maternal LG on these neuropeptide receptors, with high levels of maternal LG associated with increased OT receptors in the PVN, BNST, MPOA and CA of female offspring [Champagne et al., 2001; Francis et al., 2000, 2002], though no differences have been observed between male offspring as a function of postnatal LG
[Francis et al., 2002]. In contrast, V1a receptor levels of females do not vary in response to maternal LG whereas amongst male offspring there are increased levels of $\mathrm{Vla}$ receptors in the CA associated with high levels of maternal LG [Francis et al., 2002]. Juvenile social experiences have been found to induce changes in OT receptors in females, with social impoverishment associated with decreases in OT receptor density in the MPOA and amygdala and social enrichment associated with increased OT receptor density in these regions [Champagne and Meaney, 2007]. Changes in social group dynamics have been demonstrated to alter V1a receptor levels; following repeated aggressive encounters; dominant male Syrian hamsters show increased V1a receptor binding in the lateral VMH [Cooper et al., 2005] and in rats, dominant males have reduced V1A receptor binding in the lateral septum compared to subordinates [Askew et al., 2006]. The high degree of plasticity in OTR/V1a pathways may permit the quality of the social environment throughout development to shift brain function and behavior, with possible adaptive consequences. Though there is certainly strong evidence that this is mechanistically achieved via the epigenetic regulation of gene expression in response to early maternal behavior [Champagne, 2008; Champagne et al., 2006; Weaver et al., 2004], the importance of the weaning process for the transition of pups to an autonomous stage of development suggest that that mother-infant interactions in the later postnatal period may also be a critical cue for the establishment of stable variations in the activity of genes involved in social and reproductive behavior. Though the adaptive significance of differential weaning age in an ecological context has not been explored, it is clear that the gradual changes in the mother-infant relationship during weaning have considerable meaning within the context of development.

\section{Acknowledgement}

This research was supported by Grant Number DP2OD001674 from the Office of the Director, National Institutes of Health. 


\section{References}

Adriani W, Laviola G (2002): Spontaneous novelty seeking and amphetamine-induced conditioning and sensitization in adult mice: evidence of dissociation as a function of age at weaning. Neuropsychopharmacology 27: 225-236.

-Askew A, Gonzalez FA, Stahl JM, Karom MC (2006): Food competition and social experience effects on V1a receptor binding in the forebrain of male Long-Evans hooded rats. Horm Behav 49:328-336.

- Bale TL, Davis AM, Auger AP, Dorsa DM, McCarthy MM (2001): CNS region-specific oxytocin receptor expression: importance in regulation of anxiety and sex behavior. J Neurosci 21:2546-2552.

Benus RF, Rondigs M (1996): Patterns of maternal effort in mouse lines bidirectionally selected for aggression. Anim Behav 51:67-75.

-Berman C (1990): Intergenerational transmission of maternal rejection rates among freeranging rhesus monkeys on Cayo Santiago. Anim Behav 44:247-258.

-Bielsky IF, Hu SB, Szegda KL, Westphal H, Young LJ (2004): Profound impairment in social recognition and reduction in anxiety-like behavior in vasopressin V1a receptor knockout mice. Neuropsychopharmacology 29: 483-493.

Bielsky IF, Hu SB, Young LJ (2005): Sexual dimorphism in the vasopressin system: lack of an altered behavioral phenotype in female V1a receptor knockout mice. Behav Brain Res 164:132-136.

Birke LI, Sadler D (1987): Differences in maternal behavior of rats and the sociosexual development of the offspring. Dev Psychobiol 20:85-99.

Bosch OJ, Neumann ID (2008): Brain vasopressin is an important regulator of maternal behavior independent of dams' trait anxiety. Proc Natl Acad Sci USA 105:17139-17144

- Brunelli SA, Shindledecker RD, Hofer MA (1989): Early experience and maternal behavior in rats. Dev Psychobiol 22:295-314.

-Cameron N, Del Corpo A, Diorio J, McAllister K, Sharma S, Meaney MJ (2008a): Maternal programming of sexual behavior and hypothalamic-pituitary-gonadal function in the female rat. PLoS ONE 3:e2210.

-Cameron NM, Fish EW, Meaney MJ (2008b): Maternal influences on the sexual behavior and reproductive success of the female rat. Horm Behav 54:178-184.

-Champagne FA (2008): Epigenetic mechanisms and the transgenerational effects of maternal care. Front Neuroendocrinol 29:386-397.

-Champagne FA, Curley JP, Keverne EB, Bateson PP (2007): Natural variations in postpartum maternal care in inbred and outbred mice. Physiol Behav 91:325-334.
Champagne F, Diorio J, Sharma S, Meaney MJ (2001): Naturally occurring variations in maternal behavior in the rat are associated with differences in estrogen-inducible central oxytocin receptors. Proc Natl Acad Sci USA 98:12736-12741.

Champagne FA, Francis DD, Mar A, Meaney MJ (2003): Variations in maternal care in the rat as a mediating influence for the effects of environment on development. Physiol Behav 79:359-371.

Champagne FA, Meaney MJ (2007): Transgenerational effects of social environment on variations in maternal care and behavioral response to novelty. Behav Neurosci 121: 1353-1363.

Champagne FA, Weaver IC, Diorio J, Dymov S, Szyf M, Meaney MJ (2006): Maternal care associated with methylation of the estrogen receptor-alphalb promoter and estrogen receptor-alpha expression in the medial preoptic area of female offspring. Endocrinology 147:2909-2915.

-Cooper MA, Karom M, Huhman KL, Albers HE (2005): Repeated agonistic encounters in hamsters modulate AVP V1a receptor binding. Horm Behav 48:545-551.

Counsilman JJ, Lim LM (1985): The definition of weaning. Anim Behav 32:1023-1024

Cushing BS, Kramer KM (2005): Mechanisms underlying epigenetic effects of early social experience: the role of neuropeptides and steroids. Neurosci Biobehav Rev 29:1089-1105.

- Devidze N, Mong JA, Jasnow AM, Kow LM, Pfaff DW (2005): Sex and estrogenic effects on coexpression of mRNAs in single ventromedial hypothalamic neurons. Proc Natl Acad Sci USA 102:14446-14451.

Donaldson ZR, Young LJ (2008): Oxytocin, vasopressin, and the neurogenetics of sociality. Science 322:900-904.

Dugger BN, Morris JA, Jordan CL, Breedlove SM (2007): Androgen receptors are required for full masculinization of the ventromedial hypothalamus (VMH) in rats. Horm Behav 51: 195-201.

Egashira N, Tanoue A, Matsuda T, Koushi E, Harada S, Takano Y, Tsujimoto G, Mishima K, Iwasaki K, Fujiwara M (2007): Impaired social interaction and reduced anxiety-related behavior in vasopressin V1a receptor knockout mice. Behav Brain Res 178:123-127.

Fairbanks LA (1989): Early experience and crossgenerational continuity of mother-infant contact in vervet monkeys. Dev Psychobiol 22:669-681.

Fang J, Clemens LG (1999): Contextual determinants of female-female mounting in laboratory rats. Animal Behaviour 57:545-555.

- Ferdman N, Murmu RP, Bock J, Braun K, Leshem M (2007): Weaning age, social isolation, and gender, interact to determine adult explorative and social behavior, and dendritic and spine morphology in prefrontal cortex of rats. Behav Brain Res 180:174-182.
File SE, Seth P (2003): A review of 25 years of the social interaction test. Eur J Pharmacol 463: $35-53$.

Francis DD, Champagne FC, Meaney MJ (2000): Variations in maternal behaviour are associated with differences in oxytocin receptor levels in the rat. J Neuroendocrinol 12:11451148 .

-Francis DD, Diorio J, Liu D, Meaney MJ (1999): Nongenomic transmission across generations of maternal behavior and stress responses in the rat. Science 286:1155-1158.

sponses in the rat. Science 286:1155-1158.
Francis DD, Young LJ, Meaney MJ, Insel TR (2002): Naturally occurring differences in maternal care are associated with the expression of oxytocin and vasopressin (V1a) receptors: gender differences. J Neuroendocrinol 14:349-353.

Harding SM, McGinnis MY (2004): Androgen receptor blockade in the MPOA or VMN: effects on male sociosexual behaviors. Physiol Behav 81:671-680.

Ito A, Kikusui T, Takeuchi Y, Mori Y (2006): Effects of early weaning on anxiety and autonomic responses to stress in rats. Behav Brain Res 171:87-93.

Iwata E, Kikusui T, Takeuchi Y, Mori Y (2007): Fostering and environmental enrichment ameliorate anxious behavior induced by early weaning in Balb/c mice. Physiol Behav 91: 318-324.

Janus K (1987): Early separation of young rats from the mother and the development of play fighting. Physiol Behav 39:471-476.

Joffe JM, Levine S (1973): Effects of weaning age and adult handling on avoidance conditioning, open-field behavior, and plasma corticosterone of adult rats. Behav Biol 9:235244

Kanari K, Kikusui T, Takeuchi Y, Mori Y (2005): Multidimensional structure of anxiety-related behavior in early-weaned rats. Behav Brain Res 156:45-52.

Keverne EB, Curley JP (2004): Vasopressin, oxytocin and social behaviour. Curr Opin Neurobiol 14:777-783.

Kikusui T, Isaka Y, Mori Y (2005): Early weaning deprives mouse pups of maternal care and decreases their maternal behavior in adulthood. Behav Brain Res 162:200-206.

Kikusui T, Kiyokawa Y, Mori Y (2007): Deprivation of mother-pup interaction by early weaning alters myelin formation in male, but not female, ICR mice. Brain Res 1133:115122.

Kikusui T, Nakamura K, Kakuma Y, Mori Y (2006): Early weaning augments neuroendocrine stress responses in mice. Behav Brain Res 175:96-103.

-Kikusui T, Takeuchi Y, Mori Y (2004): Early weaning induces anxiety and aggression in adult mice. Physiol Behav 81:37-42. 
Kodama Y, Kikusui T, Takeuchi Y, Mori Y (2008): Effects of early weaning on anxiety and prefrontal cortical and hippocampal myelination in male and female Wistar rats. Dev Psychobiol 50:332-342.

Konig B, Markl H (1987): Maternal care in house mice. I. The weaning strategy as a means for parental manipulation of offspring quality. Behav Ecol Sociobiol 20:1-9.

Laviola G, Dell'Omo G (1997): Precocious weaning and changes in social variables during prepuberty affect cocaine reinforcing properties in adult mice. Psychobiology 25:163170.

Laviola G, Terranova ML (1998): The developmental psychobiology of behavioural plasticity in mice: the role of social experiences in the family unit. Neurosci Biobehav Rev 23: 197-213.

Lehmann J, Feldon J (2000): Long-term biobehavioral effects of maternal separation in the rat: consistent or confusing? Rev Neurosci 11:383-408.

- Lehmann J, Pryce CR, Bettschen D, Feldon J (1999): The maternal separation paradigm and adult emotionality and cognition in male and female Wistar rats. Pharmacol Biochem Behav 64:705-715.

-Lippmann M, Bress A, Nemeroff CB, Plotsky PM, Monteggia LM (2007): Long-term behavioural and molecular alterations associated with maternal separation in rats. Eur J Neurosci 25:3091-3098.

Maestripieri D (2005): Early experience affects the intergenerational transmission of infant abuse in rhesus monkeys. Proc Natl Acad Sci USA 102:9726-9729.

Martin P (1984): The meaning of weaning. Anim Behav 32:1257-1259.
McCarthy MM, Kleopoulos SP, Mobbs CV, Pfaff DW (1994): Infusion of antisense oligodeoxynucleotides to the oxytocin receptor in the ventromedial hypothalamus reduces estrogen-induced sexual receptivity and oxytocin receptor binding in the female rat. Neuroendocrinology 59:432-440.

Meaney MJ (2001): Maternal care, gene expres sion, and the transmission of individual differences in stress reactivity across generations. Annu Rev Neurosci 24:1161-1192.

Miller L, Kramer R, Warner V, Wickramaratne P, Weissman M (1997): Intergenerational transmission of parental bonding among women. J Am Acad Child Adolesc Psychiatry 36:1134-1139.

Moore CL (1984): Maternal contributions to the development of masculine sexual behavior in laboratory rats. Dev Psychobiol 17:347356.

Dakamura K, Kikusui T, Takeuchi Y, Mori Y (2003): The influence of early weaning on aggressive behavior in mice. J Vet Med Sci 65: 1347-1349.

Nakamura K, Kikusui T, Takeuchi Y, Mori Y (2008): Changes in social instigation- and food restriction-induced aggressive behaviors and hippocampal 5HT1B mRNA receptor expression in male mice from early weaning. Behav Brain Res 187:442-448.

Nelson RJ, Trainor BC (2007): Neural mechanisms of aggression. Nat Rev Neurosci 8: 536-546.

Nephew BC, Bridges RS (2008): Central actions of arginine vasopressin and a V1a receptor antagonist on maternal aggression, maternal behavior, and grooming in lactating rats. Pharmacol Biochem Behav 91:77-83.

Paxinos G, Franklin KBJ (2003): The Mouse Brain in Stereotaxic Coordinates. New York, Academic Press.
Shimozuru M, Kodama Y, Iwasa T, Kikusui T, Takeuchi Y, Mori Y (2007): Early weaning decreases play-fighting behavior during the postweaning developmental period of $\mathrm{Wi}$ star rats. Dev Psychobiol 49:343-350.

- Skolnick NJ, Ackerman SH, Hofer MA, Weiner $H$ (1980): Vertical transmission of acquired ulcer susceptibility in the rat. Science 208: 1161-1163.

Smith EFS (1991): Early social development in hooded rats (Rattus norvegicus): a link between weaning and play. Anim Behav 41: 513-524.

Sroufe LA (2005): Attachment and development: a prospective, longitudinal study from birth to adulthood. Attach Hum Dev 7:349-367.

Terranova ML, Laviola G (1995): Individual differences in mouse behavioural development: effects of precocious weaning and ongoing sexual segregation. Anim Behav 50:12611271.

Terranova ML, Laviola G (2001): Delta-opioid modulation of social interactions in juvenile mice weaned at different ages. Physiol Behav 73:393-400.

Terranova ML, Laviola G, de Acetis L, Alleva E (1998): A description of the ontogeny of mouse agonistic behavior. J Comp Psychol 112:3-12.

-Weaver IC, Cervoni N, Champagne FA, D’Alessio AC, Sharma S, Seckl JR, Dymov S, Szyf M, Meaney MJ (2004): Epigenetic programming by maternal behavior. Nat Neurosci 7:847854.

Windle RJ, Gamble LE, Kershaw YM, Wood SA, Lightman SL, Ingram CD (2006): Gonadal steroid modulation of stress-induced hypothalamo-pituitary-adrenal activity and anxiety behavior: role of central oxy tocin. Endocrinology 147:2423-2431. 